\title{
Hamiltonicity of minimum distance graphs of 1-perfect codes
}

\author{
Alexander M. Romanov* \\ Sobolev Institute of Mathematics \\ 630090 Novosibirsk, Russia \\ rom@math.nsc.ru
}

Submitted: Jan 18, 2012; Accepted: Mar 18, 2012; Published: Mar 31, 2012

Mathematics Subject Classifications: 05C45, 94B25

\begin{abstract}
A 1-perfect code $\mathcal{C}_{q}^{n}$ is called Hamiltonian if its minimum distance graph $G\left(\mathcal{C}_{q}^{n}\right)$ contains a Hamiltonian cycle. In this paper, for all admissible lengths $n \geq 13$, we construct Hamiltonian nonlinear ternary 1-perfect codes, and for all admissible lengths $n \geq 21$, we construct Hamiltonian nonlinear quaternary 1-perfect codes. The existence of Hamiltonian nonlinear $q$-ary 1-perfect codes of length $N=q n+1$ is reduced to the question of the existence of such codes of length $n$. Consequently, for $q=p^{r}$, where $p$ is prime, $r \geq 1$ there exist Hamiltonian nonlinear $q$-ary 1-perfect codes of length $n=\left(q^{m}-1\right) /(q-1), m \geq 2$. If $q=2,3,4$, then $m \neq 2$. If $q=2$, then $m \neq 3$.
\end{abstract}

\section{Introduction}

Let $\mathbf{F}_{q}^{n}$ be a vector space of dimension $n$ over the Galois field $\mathbf{F}_{q}$. The Hamming distance between two vectors $\mathbf{x}, \mathbf{y} \in \mathbf{F}_{q}^{n}$ is the number of coordinates in which they differ and it is denote by $d(\mathbf{x}, \mathbf{y})$. An arbitrary subset $\mathcal{C}_{q}^{n}$ of $\mathbf{F}_{q}^{n}$ is called $q$-ary 1-perfect code of length $n$, if for every vector $\mathbf{x} \in \mathbf{F}_{q}^{n}$ there exists a unique vector $\mathbf{c} \in \mathcal{C}_{q}^{n}$ such that $d(\mathbf{x}, \mathbf{c}) \leq 1$. It is known that $q$-ary 1-perfect codes of length $n$ exist only if $n=\left(q^{m}-1\right) /(q-1)$, where $m$ is a natural number not less than two. We shall assume that the all-zero vector $\mathbf{0}$ is in code. A code is called linear if it is a linear space over $\mathbf{F}_{q}$. The linear 1-perfect codes are called Hamming codes.

The sum of the vectors $\mathbf{x}, \mathbf{y} \in \mathbf{F}_{q}^{n}$ is denoted by $\mathbf{x}+\mathbf{y}$. Two codes $\mathcal{C}_{q}^{n}, \mathcal{D}_{q}^{n} \subseteq \mathbf{F}_{q}^{n}$ are said to be isomorphic if there exists a permutation $\pi$ such that $\mathcal{D}_{q}^{n}=\left\{\pi(\mathbf{c}): \mathbf{c} \in \mathcal{C}_{q}^{n}\right\}$. They are said to be equivalent if there exist a vector $\mathbf{u} \in \mathbf{F}_{q}^{n}$ and a permutation $\pi$ such

\footnotetext{
*The work is supported by RFFI (grant 11-01-00997)
} 
that $\mathcal{D}_{q}^{n}=\left\{\pi(\mathbf{c})+\mathbf{u}: \mathbf{c} \in \mathcal{C}_{q}^{n}\right\}$. There exist at least $q^{q^{c n}}$ pairwise nonequivalent $q$-ary 1-perfect codes of length $n$ where a constant $c=\frac{1}{q}-\varepsilon$. See $[1,2,6,7]$.

The minimum distance of a code $\mathcal{C}_{q}^{n} \subseteq \mathbf{F}_{q}^{n}$ is defined by $d\left(\mathcal{C}_{q}^{n}\right)=\min \{d(\mathbf{x}, \mathbf{y}): \mathbf{x}, \mathbf{y} \in$ $\left.\mathcal{C}_{q}^{n}, \mathbf{x} \neq \mathbf{y}\right\}$. Let $\mathcal{C}_{q}^{n}$ be a 1-perfect code of length $n$. Then, $d\left(\mathcal{C}_{q}^{n}\right)=3$ and the minimum distance graph of the code $\mathcal{C}_{q}^{n}$ is a graph $G\left(\mathcal{C}_{q}^{n}\right)$ whose vertex set is $\mathcal{C}_{q}^{n}$ and vertices $\mathbf{x}, \mathbf{y} \in \mathcal{C}_{q}^{n}$ are adjacent if and only if $d(\mathbf{x}, \mathbf{y})=3$. A path in a graph is a sequence of vertices such that two consecutive vertices in this path are connected by at least one edge. A finite path always has a first vertex, called its start vertex, and a last vertex, called its end vertex. A cycle is a path such that the start vertex and end vertex are the same. A cycle that contains each vertex of the graph exactly once is called Hamiltonian cycle. A 1-perfect code $\mathcal{C}_{q}^{n}$ is called Hamiltonian if its minimum distance graph $G\left(\mathcal{C}_{q}^{n}\right)$ contains a Hamiltonian cycle.

The weight of a vector $\mathbf{x} \in \mathbf{F}_{q}^{n}$ is the number of its nonzero coordinates. A vector of weight 3 of the Hamming code $\mathcal{H}_{q}^{n}$ is called triple. It is known that the set of all triples of the code $\mathcal{H}_{q}^{n}$ generates the code. Therefore, the Hamming codes are Hamiltonian, except $q=2, m=2$.

A mapping $\phi: \mathcal{C}_{q}^{n} \rightarrow \mathbf{F}_{q}^{n}$ is called an isometry from the code $\mathcal{C}_{q}^{n}$ to the code $\phi\left(\mathcal{C}_{q}^{n}\right)$ if $d(\mathbf{x}, \mathbf{y})=d(\phi(\mathbf{x}), \phi(\mathbf{y}))$ for all $\mathbf{x}, \mathbf{y} \in \mathcal{C}_{q}^{n}$. Obviously, two codes $\mathcal{C}_{q}^{n}$ and $\mathcal{D}_{q}^{n}$ are isometric if there are $n$ permutations $\tau_{1}, \tau_{2}, \ldots, \tau_{n}$ of $q$ elements in Galois field $\mathbf{F}_{q}$ and permutation $\sigma$ of the $n$ coordinates such that $\mathcal{D}_{q}^{n}=\left\{\sigma\left(\tau_{1}\left(c_{1}\right), \tau_{2}\left(c_{2}\right), \ldots, \tau_{n}\left(c_{n}\right)\right): \mathbf{c}=\left(c_{1}, c_{2}, \ldots, c_{n}\right) \in \mathcal{C}_{q}^{n}\right\}$.

For $q \geq 5$ there are nonlinear $q$-ary 1-perfect codes that are isometric to the Hamming codes. Therefore, for $q \geq 5$ and for all admissible lengths there are nonlinear $q$-ary 1-perfect codes whose minimum distance graphs contain a Hamiltonian cycle.

The question of the existence of Hamiltonian nonlinear binary 1-perfect codes remained an open for a long time. The existence of Hamiltonian nonlinear binary codes for all admissible lengths $n \geq 15$ was constructively proved in [4].

In this paper, for all admissible lengths $n \geq 13$, we construct Hamiltonian nonlinear ternary 1-perfect codes, and for all admissible lengths $n \geq 21$, we construct Hamiltonian nonlinear quaternary 1-perfect codes. The existence of Hamiltonian nonlinear q-ary 1perfect codes of length $N=q n+1$ is reduced to the question of the existence of such codes of length $n$. Consequently, for $q=p^{r}$, where $p$ is prime, $r \geq 1$ there exist Hamiltonian nonlinear $q$-ary 1-perfect codes of length $n=\left(q^{m}-1\right) /(q-1), m \geq 2$. If $q=2,3,4$, then $m \neq 2$. If $q=2$, then $m \neq 3$.

It remains an open question of the existence of a Hamiltonian cycle in the graph formed by the two middle levels of $n$-dimensional binary hypercube, where $n$ is odd. Also, is not proved Lovász conjecture, which states that the every finite connected vertex-transitive graph contains a Hamiltonian path.

Let $\mathcal{H}_{q}^{n}$ be a $q$-ary Hamming code of length $n$. The parity-check matrix of Hamming code $\mathcal{H}_{q}^{n}$ of length $n=\left(q^{m}-1\right) /(q-1)$ consists of $n$ pairwise linearly independent column vectors $\mathbf{h}_{i}$, where $\mathbf{h}_{i}^{T} \in \mathbf{F}_{q}^{m}, i \in\{1, \ldots, n\}$. The set $\mathbf{F}_{q}^{m} \backslash\{\mathbf{0}\}$ generates a projective space $P G_{m-1}(q)$ of dimension $(m-1)$ over the Galois field $\mathbf{F}_{q}$. In this space, points correspond to the column vectors of the parity-check matrix of the Hamming code $\mathcal{H}_{q}^{n}$ and the three points $i, j, k$ lie on the same line if the corresponding column vectors $\mathbf{h}_{\mathbf{i}}, \mathbf{h}_{\mathbf{j}}, \mathbf{h}_{\mathbf{k}}$ are linearly 
dependent.

Let $\mathbf{x}=\left(x_{1}, x_{2}, \ldots, x_{n}\right) \in \mathbf{F}_{q}^{n}$. Then, the support of vector $\mathbf{x}$ is the set $\operatorname{supp}(\mathbf{x})=$ $\left\{i: x_{i} \neq 0\right\}$. Consider the vector $\mathbf{x} \in \mathbf{F}_{q}^{n}$ such that its $\operatorname{supp}(\mathbf{x})$ is $m-2$ dimensional hyperplane. Denote by $\mathcal{H}_{q}^{n}(\mathbf{x})$ the set of all vectors $\mathbf{v} \in \mathcal{H}_{q}^{n}$ such that $\operatorname{supp}(\mathbf{v}) \subseteq \operatorname{supp}(\mathbf{x})$. The set $\mathcal{H}_{q}^{n}(\mathbf{x})$ forms in $\mathcal{H}_{q}^{n}$ subcode previous dimension.

Let $N=q n+1=\left(q^{m+1}-1\right) /(q-1)$. In the projective space $P G_{m}(q)$ of dimension $m$, we consider the pencil of lines through a point $i$, where $i \in\{1,2, \ldots, N\}$. It is known that the pencil of lines contains $n$ lines which will be denoted by $l_{1}, l_{2}, \ldots, l_{n}$. Denote by $\mathcal{H}_{q}^{N}\left(l_{p}\right)$ the subcode of the code $\mathcal{H}_{q}^{N}$ defined by the line $l_{p}, p \in\{1,2, \ldots, n\}$. Let

$$
R_{i}^{N}=\mathcal{H}_{q}^{N}\left(l_{1}\right)+\mathcal{H}_{q}^{N}\left(l_{2}\right)+\cdots+\mathcal{H}_{q}^{N}\left(l_{n}\right) .
$$

All cosets $R_{i}^{N}+\mathbf{v}$ (where $\mathbf{v} \in \mathcal{H}_{q}^{N}$ ) form the set of $i$-components of the Hamming code $\mathcal{H}_{q}^{N}$. Since the dimension of $\mathcal{H}_{q}^{N}\left(l_{p}\right)$ is $q-1$, it follows that the dimension of $R_{i}^{N}$ is $(q-1) n=q^{m}-1$. See $[3,5]$.

A triple belongs to the line if the support of this triple belongs to the line. Consider the subspace $R_{i}^{n}$ of the Hamming code $\mathcal{H}_{q}^{n}$ of length $n$. In the projective space $P G_{m-1}(q)$, each line contains $q+1$ points. Therefore, each line contains $q-1$ linearly independent triples with nonzero $i$ th coordinate which form the basis of the subspace $\mathcal{H}_{q}^{n}\left(l_{p}\right)$, where $p \in\{1, l, 2, \ldots,(n-1) / q\}$. Thus the subspace $R_{i}^{n}$ is generated by all triples with nonzero $i$ th coordinate. The dimension of $R_{i}^{n}$ is $q^{m-1}-1$. Let $\mathbf{e}_{i}$ denote the vector of length $n$ in which $i$ th component is 1 and other components are equal to 0 . Let $\lambda \in \mathbf{F}_{q}$. It is known [3], the set

$$
\mathcal{C}_{q}^{n}=\left(\mathcal{H}_{q}^{n} \backslash\left(R_{i}^{n}+\mathbf{u}\right)\right) \cup\left(R_{i}^{n}+\mathbf{u}+\lambda \cdot \mathbf{e}_{i}\right)
$$

is a 1-perfect code of length $n$. It is said that the code $\mathcal{C}_{q}^{n}$ is obtained from the code $\mathcal{H}_{q}^{n}$ by switching or translation of the $i$-component $\left(R_{i}^{n}+\mathbf{u}\right)$ of the code $\mathcal{H}_{q}^{n}$.

\section{Main results}

Consider the vector $\mathbf{x} \in \mathbf{F}_{q}^{n}$ such that its $\operatorname{supp}(\mathbf{x})$ is $m-2$ dimensional hyperplane. Denote by $\mathbf{F}_{q}^{n}(\mathbf{x})$ the set of all vectors $\mathbf{v} \in \mathbf{F}_{q}^{n}$ such that $\operatorname{supp}(\mathbf{v}) \subseteq \operatorname{supp}(\mathbf{x})$.

Lemma 1. Let $i \notin \operatorname{supp}(\mathbf{x})$ and $\mathbf{u} \in \mathbf{F}_{q}^{n}(\mathbf{x})$. Then, the intersection

$$
\left(R_{i}^{n}+\mathbf{u}\right) \cap \mathbf{F}_{q}^{n}(\mathbf{x})
$$

contains only one vector.

Proof. Let $l_{p}$ be an arbitrary line through the point $i$. Since $i \notin \operatorname{supp}(\mathbf{x})$, it follows that any line passing through the point $i$ intersects with the hyperplane $\operatorname{supp}(\mathbf{x})$ only at one point. Hence the intersection of $\mathcal{H}_{q}^{n}\left(l_{p}\right) \cap \mathbf{F}_{q}^{n}(\mathbf{x})$ can contain only vectors of weight 0 or 1 . It is obvious that $\mathbf{0} \in \mathcal{H}_{q}^{n}\left(l_{p}\right) \cap \mathbf{F}_{q}^{n}(\mathbf{x})$. Since the minimum weight of the nonzero vectors in $\mathcal{H}_{q}^{n}\left(l_{p}\right)$ is equal to 3 , it follows that $\mathcal{H}_{q}^{n}\left(l_{p}\right) \cap \mathcal{R}_{q}^{n}(\mathbf{x})=\{\mathbf{0}\}$. Since the line $l_{p}$ was chosen arbitrarily and set $R_{i}^{n}$ is a subspace, we have $R_{i}^{n} \cap \mathcal{R}_{q}^{n}(\mathbf{x})=\{\mathbf{0}\}$. The lemma is proved. 
Obvious that the minimum distance between two distinct vectors in $R_{i}^{n}$ is equal to 3 . Denote by $G\left(R_{i}^{n}\right)$ the minimum distance graph of the set $R_{i}^{n}$.

Lemma 2. Let $m \geq 3, n=\left(q^{m}-1\right) /(q-1)$. Then, graph $G\left(R_{i}^{n}\right)$ contains a Hamiltonian cycle.

Proof. Consider a cyclic $q$-ary Gray code of dimension $q^{m-1}-1$. The Gray code defines a linear combinations of the basis vectors of the subspace $R_{i}^{n}$. Since the subspace $R_{i}^{n}$ is generated by all triples with non-zero $i$ th coordinate, it follows that all basis vectors of the subspace $R_{i}^{n}$ have weight equal to the 3 . Hamming distance between two consecutive vectors in the Gray code is one. Therefore, the distance between two consecutive vectors (the consecutive vectors in the subspace $R_{i}^{n}$ defined by the consecutive vectors of the Gray code) in the subspace $R_{i}^{n}$ is 3 . Thus a cyclic $q$-ary Gray code of dimension $q^{m-1}-1$ determines a Hamiltonian cycle in the graph $G\left(R_{i}^{n}\right)$. The lemma is proved.

Now we turn to a proof of the main theorem.

Theorem 1. Assume that there exists a nonlinear q-ary 1-perfect code $\mathcal{C}_{q}^{n}$ of length $n=\left(q^{m-1}-1\right) /(q-1), m \geq 3$ such that the minimum distance graph of the code $\mathcal{C}_{q}^{n}$ contains a Hamiltonian cycle. Then, there exists a nonlinear q-ary 1-perfect code $\mathcal{D}_{q}^{N}$ of length $N=q n+1$ such that the minimum distance graph of the code $\mathcal{D}_{q}^{N}$ also contains a Hamiltonian cycle.

Proof. Consider the construction of nonlinear $q$-ary 1-perfect codes proposed in $[2,6]$. This construction is a generalization of the construction from [7]. We assume that the columns of parity-check matrix of the Hamming code $\mathcal{H}_{q}^{N}$ are ordered lexicographically. The vectors of the space $\mathbf{F}_{q}^{N}$ will also be considered as words of length $N$ over an alphabet $\{0,1, \ldots, q-1\}$. Let

$$
\mathcal{D}_{q}^{N}=\bigcup_{\mathbf{c} \in \mathcal{C}_{q}^{n}}\left(R_{i}^{N}+(\mathbf{c} \mid \mathbf{0})\right)
$$

where the vector $\mathbf{0} \in \mathbf{F}_{q}^{(q n-n+1)}, i \geq n+1$ and the vertical bar $(\mid)$ denotes concatenation. Formula (1) is a certain modification of the construction from $[2,6]$. Lemma 1 implies that the set $\mathcal{D}_{q}^{N}$ is a $q$-ary 1 -perfect code of length $N=q n+1$. The nonlinearity of the code $\mathcal{D}_{q}^{N}$ follows from (1) and the nonlinearity of the code $\mathcal{C}_{q}^{n}$. It is known that the graph that is a Cartesian product of two Hamiltonian cycles always contains a Hamiltonian cycle. Consequently, hamiltonicity of code $\mathcal{D}_{q}^{N}$ follows from Lemma 2, formula (1) and hamiltonicity of the code $\mathcal{C}_{q}^{n}$. The theorem is proved.

Statement 1. Let $i \notin \operatorname{supp}(\mathbf{x})$ and $\mathbf{u} \in \mathcal{H}_{q}^{n}$. Then, the intersection

$$
\left(R_{i}^{n}+\mathbf{u}\right) \cap \mathcal{H}_{q}^{n}(\mathbf{x})
$$

contains only one vector.

Proof. Let $l_{p}$ be an arbitrary line through the point $i$. Since $i \notin \operatorname{supp}(\mathbf{x})$, it follows that any line passing through the point $i$ intersects with the hyperplane $\operatorname{supp}(\mathbf{x})$ only at one 
point. Hence, the intersection of $R_{i}^{n} \cap \mathcal{H}_{q}^{n}(\mathbf{x})$ can contain only vectors of weight 0 or 1 . It is obvious that $\mathbf{0} \in \mathcal{H}_{q}^{n}\left(l_{p}\right) \cap \mathcal{H}_{q}^{n}(\mathbf{x})$. Since the minimum weight of nonzero vectors that belong to the subcode $\mathcal{H}_{q}^{n}\left(l_{p}\right)$ is 3 , it follows that $\mathcal{H}_{q}^{n}\left(l_{p}\right) \cap \mathcal{H}_{q}^{n}(\mathbf{x})=\{\mathbf{0}\}$. Since the line $l_{p}$ was chosen arbitrarily and set $R_{i}^{n}$ is a subspace, we have $R_{i}^{n} \cap \mathcal{H}_{q}^{n}(\mathbf{x})=\{\mathbf{0}\}$. Next, we show that the number of cosets formed subspace $R_{i}^{n}$ equals the number code vectors belonging to the subcode $\mathcal{H}_{q}^{n}(\mathbf{x})$. The dimension of the code $\mathcal{H}_{q}^{n}$ is equal to $n-m$, and the dimension of $R_{i}^{n}$ is $q^{m-1}-1$. Hence, the number of cosets formed subspace $R_{i}^{n}$ is equal to $q^{\frac{n-1}{q}-m+1}$. Number of codewords in the subcode $\mathcal{H}_{q}^{n}(\mathbf{x})$ is equal to $q^{\frac{n-1}{q}-m+1}$. Thus we have the equality $\left|\left(R_{i}^{n}+\mathbf{u}\right) \cap \mathcal{H}_{q}^{n}(\mathbf{x})\right|=1$. The statement is proved.

Statement 2. Let $N=q n+1=\left(q^{m+1}-1\right) /(q-1)$. Then, the graph $G\left(\mathcal{H}_{q}^{N}\right)$ contains a spanning subgraph which is a Cartesian product of a Hamiltonian cycle of the graph $G\left(\mathcal{H}_{q}^{n}\right)$ and a Hamiltonian cycle of the graph $G\left(R_{i}^{N}\right)$.

Proof. Let $i \notin\{1,2, \ldots, n\}$. Then, by Statement 1 for the Hamming code $\mathcal{H}_{q}^{N}$ we have

$$
\mathcal{H}_{q}^{N}=\bigcup_{\mathbf{c} \in \mathcal{H}_{q}^{n}}\left(R_{i}^{N}+(\mathbf{c} \mid \mathbf{0})\right) .
$$

Hence, the graph $G\left(\mathcal{H}_{q}^{N}\right)$ contains a spanning subgraph which is a Cartesian product of a Hamiltonian cycle of the graph $G\left(\mathcal{H}_{q}^{n}\right)$ and a Hamiltonian cycle of the graph $G\left(R_{i}^{N}\right)$. The statement is proved.

Next, let $q=3$. For $m=2$ all ternary 1-perfect codes of length $n=4$ are equivalent to the ternary Hamming code $\mathcal{H}_{3}^{4}$. A minimum distance graph of the ternary Hamming code $\mathcal{H}_{3}^{4}$ is a complete graph on nine vertices.

Theorem 2. For $q=3$ and $q=4, m \geq 3$ there exist nonlinear $q$-ary 1-perfect codes of length $n=\left(q^{m-1}-1\right) /(q-1)$ whose minimum distance graphs contain a Hamiltonian cycle.

Proof. We can construct the nonlinear ternary 1-perfect codes of length $n=13$ by switching of $i$-components of the ternary Hamming code $\mathcal{H}_{3}^{13}$ and inspect by computer that the minimum distance graphs of these codes contain a Hamiltonian cycle. If we use Statement 2, then the construction of Hamiltonian nonlinear ternary 1-perfect codes of length $n=13$ will be simple. Similarly we can construct the Hamiltonian nonlinear quaternary 1-perfect codes of length $n=21$. The theorem is proved.

\section{References}

[1] T. Etzion Nonequivalent q-ary Perfect Codes. SIAM J. Disc. Math. 9 (1996) 413-423.

[2] B. Lindström On group and nongroup perfect codes in $q$ symbols. Math. Scand. 25 (1969) 149-158.

[3] K. T. Phelps, M. Villanueva Ranks of q-ary 1-perfect codes. Des. Codes Cryptogr. 27 (2002) 139-144. 
[4] A. M. Romanov On combinatorial Gray codes with distance 3. Disc. Math. Appl. 19 (2009) 383-388.

[5] A. M. Romanov On partitions of q-ary Hamming codes into disjoint components. Diskretn. Anal. Issled. Oper. Ser. 1, 11, (2004) 80-87.

[6] J.Schönheim On linear and nonlinear single-error-correcting q-nary perfect codes. Inform. and Control 12 (1968) 23-26.

[7] Yu. L. Vasil'ev On nongroup close-packed codes. Probl. Kybern. 8 (1962) 337-339. 